\title{
LETTER TO THE EDITOR Early Diagnosis Remains the Most Reliable Way to Cure Chidren With Melanoma: Response
}

We thank Drs. Aldo and Ferrari for sharing their clinical experience with pediatric melanoma. They make several important points in their letter which we feel require emphasis. The clinical characteristics of pediatric melanomas often do not follow the "ABCD rule" classically seen in adult melanomas. In fact, childhood melanomas are frequently amelanotic and often lack particularly worrisome features, characteristics that may contribute to delays in diagnosis, resulting in more advanced disease and worse prognosis. These atypical features associated with pediatric melanomas therefore make it difficult to have a well-founded clinical suspicion of malignancy. It is precisely because the diagnosis of pediatric melanoma can be difficult that prompt referral to a dermatologist or pediatric oncologist with experience in the diagnosis and management of pediatric melanoma is warranted for any suspected melanocytic skin lesion. We certainly agree that the avoidance of patient/parent stress and conservation of medical resources are worthwhile goals. However, because early detection offers the best chance of cure for melanoma patients, we would be concerned about a "short-term" observation period, and would consider careful and limited excisional biopsy of suspected melanocytic lesions in children sooner rather than later. Together with our report, the observations of Drs. Aldo and Ferrari should reinforce to primary caregivers and parents that the incidence of malignant melanoma is increasing in Western countries, and that these tumors do occur in children who are not otherwise at risk.

Mwe Mwe Chao, MD* Daniel S. Wechsler, MD, PhD Section of Pediatric Hematology-Oncology The University of Michigan Health System Ann Arbor, Michigan
*Correspondence to: Mwe Mwe Chao, Section of Pediatric Hematology-Oncology, The University of Michigan Health Systems, Ann Arbor, MI 48109-0936. E-mail: mmchao@umich.edu

Received 2 February 2005; Accepted 3 February 2005 